\section{Effect Analysis of Hyperbaric Oxygen Therapy with Methylprednisolone on Prevention of Spinal Cord Ischemia-Reperfusion Injury}

Sir,

Patients with cervical and thoracic spinal stenosis generally have a good recovery of spinal cord function after spinal canal decompressive operation. However, after the removal of some factors leading to spinal cord ischemia, the blood supply of the spinal cord is restored. The neurological function of some patients are not improved, but are aggravated on the basis of original ischemic injury, and even the phenomenon of irreversible delayed death of spinal cord neurons occurs. This phenomenon is called Spinal Cord Ischemia-Reperfusion Injury (SCIRI). ${ }^{1}$ It is a secondary injury after primary spinal cord injury, and an important factor causing nerve cell injury. It can cause quadriplegia, paraplegia and even death. Therefore, it is particularly important to strengthen the prevention and treatment of SCIRI. Methylprednisolone (MP) is a synthetic glucocorticoid, which can increase spinal cord blood flow, reduce lipid peroxidation and tissue degeneration of spinal cord. 2 Studies have shown that hyperbaric oxygen can increase the oxygen tension after spinal cord injury, make tissue repair and restore nerve function. ${ }^{3}$ In recent years, the author has observed that hyperbaric oxygen therapy combined with methylprednisolone can effectively prevent SCIRI.

A total of 186 patients with cervical and thoracic spinal stenosis (the causes of thoracic spinal stenosis were ligamenta flava thickening or ossify) received and managed in West China Second Hospital, Sichuan University, China, were selected as the research subjects. Complex injuries such as traumatic brain injury, thoracic and abdominal organ injury, combined with tumors and surgical contraindication were excluded. This study was approved by the hospital ethics committee. All patients were treated with posterior extensive laminectomy decompression and thorough decompression of posterior epidural side. All operated by a certified senior surgeon. According to a random number table method, the patients were randomly divided into group $A$ and group $B$, with 93 cases in each group. Group A was treated with $0.1 \mathrm{MPa}$ hyperbaric oxygen (once a hour, once a day) for one week before operation and for 7 days from 3 days after operation. At the same time, $1000 \mathrm{mg}$ of MP was intravenously dripped 30 minutes before decompression, and finished within 15 minutes. The daily MP dosage decreased by $200 \mathrm{mg}$ from the first day after decompression, and the drug was stopped on the fifth day after decompression. Group B was treated with $20 \mathrm{mg}$ of dexamethasone intravenously before operation. After 7 days of continuous intravenous drip, Group B took dexamethasone orally three times a day, $0.75 \mathrm{mg}$ each time for 14 days. Data was analysed in SPSS version 21 . The Chi-square test was applied to compare the Groups in incidence rate of ischemia-reperfusion injury. The results were considered significant at $p<0.05$.

Among 186 patients, there were 107 males (57.53\%) and 79 females $(42.47 \%)$. The age ranged from 34 to 58 with an average age $47.15 \pm 2.83$ years. The course of disease ranged from 7 months to 9 years with an average duration $2.36 \pm 0.42$ years. Preoperative American Spinal Injury Association (ASIA) Grade E was $146(78.49 \%)$ cases, ASIA Grade D was $40(21.51 \%)$ cases.

At 2-4 hours after decompressive surgery, 1 case $(1.08 \%)$ was ASIA Grade A in Group A and 8 cases $(8.60 \%)$ were ASIA Grade A in Group B; and all of them suddenly showed progressive decline to disappearance of sensation and movement below the decompression level, and all kinds of physiological reflexes of both lower extremities and preoperative pathological reflexes disappeared, similar to the manifestations of spinal cord shock. The spinal cord compression caused by mechanical factors such as postoperative hematoma and the lesions of spinal cord and brain above decompression level were excluded by imaging and related examinations, and the spinal cord ischemiareperfusion injury after decompressive surgery was considered. There was a significant difference in the incidence rate of ischemia-reperfusion injury between the two groups $(p=0.017)$. The patients with ischemiareperfusion injury recovered within 2 weeks after further treatment in both Groups.

The application of hyperbaric oxygen therapy combined with MP before and after spinal canal decompression surgery for cervical and thoracic spinal stenosis is superior to that of dexamethasone in preventing the spinal cord ischemia reperfusion injury. The change of microcirculation is one of the important mechanisms of ischemia-reperfusion injury. In animal experiments Kahraman et al. found that hyperbaric oxygen seemd to procure prevention against oxidative spinal cord injury. 4 MP can inhibit inflammation, reduce edema, inhibit vascular activity and prostaglandin activity, and increase spinal cord blood flow. ${ }^{5}$ Hyperbaric oxygen therapy combined with MP can play a synergistic role in promoting the recovery of spinal cord function. 


\section{CONFLICT OF INTEREST:}

Authors declared no conflict of interest.

AUTHORS' CONTRIBUTION:

YC: Responsible for the conception or design of the work; drafting the work.

ZF: Responsible for acquisition, analysis, and interpretation of data for the work.

LL: Responsible for the acquisition of data for the work; drafting the work.

YL: Revised it critically for important intellectual content; final approval of the version to be published.

\section{REFERENCES}

1. Mongardon N, Kohlhauer M, Lidouren F, Barretto M, Micheau P, Adam $C$, et al. Targeted temperature management with total liquid ventilation after ischemic spinal cord injury. Ann Thorac Surg 2018; 106:1797-803.

2. Chen XG, Chen LH, Xu RX, Zhang HT. Effect evaluation of methylprednisolone plus mitochondrial division inhibitor-1 on spinal cord injury rats. Childs Nerv Syst 2018; 34:1479-87.

3. Hentia C, Rizzato A, Camporesi E, Yang Z, Muntean DM, Sandesc $D$, et al. An overview of protective strategies against ischemia/reperfusion injury: The role of hyperbaric oxygen preconditioning. Brain Behav 2018; 8:e00959.

4. Kahraman S, Düz B, Kayali H, Korkmaz A, Oter S, Aydin A, et al. Effects of methylprednisolone and hyperbaric oxygen on oxidative status after experimental spinal cord injury: A comparative study in rats. Neurochem Res 2007; 32:1547-51.

5. Visna P, Wendsche P, Svoboda P, Kantorová I. The response of methylprednisolon therapy to interleukin-6 release in spinal injuries. Acta Chir Orthop Traumatol Cech 1998; 65:207-10.

Yuan Chen', Ziyan Fan'2, Lingyi Liao ${ }^{2}$ and Yunzhu Lin ${ }^{1}$

1 Department of Pharmacy, Evidence-based Pharmacy Center, West China Second Hospital, Sichuan University, Chengdu, Sichuan Province, 610041, China

2 Department of Rehabilitation Medicine, West China Hospital, Sichuan University, Sichuan, 610041, China

Correspondence to: Yunzhu Lin, Department of Pharmacy, Evidence-based Pharmacy Center, West China Second Hospital, Sichuan University, Chengdu, Sichuan Province, 610041, China E-mail:mkgix1@163.com

Received: November 26, 2018; Revised: February 02, 2019; Accepted: March 18, 2019 